\title{
NITROGÊNIO E POTÁSSIO NO CRESCIMENTO DE MUDAS DE PITAIA [Hylocereus undatus (Haw.) BRITTON \& ROSE] ${ }^{1}$
}

\author{
EDMILSON IGOR BERNARDO ALMEIDA², MÁRCIO CLEBER DE MEDEIROS CORRÊA ${ }^{3}$, \\ LINDBERGUE ARAUJO CRISOSTOMO ${ }^{4}$, NEIMAR ARCANJO DE ARAÚJO 5 , \\ JÚLIO CÉSAR DO VALE SILVA 6
}

RESUMO - A pitaia ainda é uma cultura carente de estudos e informações que subsidiem a definição/ adaptação de sistemas de produção mais adequados às condições edafoclimáticas brasileiras, embora nos últimos anos tenha havido aumento de sua expansão agrícola, tanto no Brasil quanto em outros países. Assim, o objetivo deste estudo foi avaliar o efeito da adubação mineral sobre o crescimento de mudas de pitaia e, dessa maneira, obter informações direcionadas para a definição de manejos de fertilização adequados à exploração agrícola da cultura. Para isto, foram testadas cinco doses de nitrogênio (N) $(0 ; 150 ; 300 ; 450$ e $\left.600 \mathrm{mg} \mathrm{dm}^{-3}\right)$ e potássio $(\mathrm{K})\left(0 ; 75 ; 150 ; 225\right.$ e $\left.300 \mathrm{mg} \mathrm{dm}^{-3}\right)$, sendo os níveis de cada nutriente arranjados em esquema fatorial $5 \times 5$, com quatro blocos. Para a interação significativa a $(\mathrm{p}<0,05)$, procedeu-se ao ajuste em superfícies de resposta do tipo $Y=\beta_{0}+\beta_{1}(\mathrm{~A})+\beta_{2}(\mathrm{~A})^{2}+\beta_{3}(\mathrm{~B})+\beta_{4}(\mathrm{~B})^{2}+\beta_{5}(\mathrm{~A})$. (B) $+e$. As estimativas das correlações fenotípicas foram obtidas considerando apenas o intervalo das doses de $\mathrm{N}$ e $\mathrm{K}$, que promoveram as melhores respostas do acesso de pitaia para os caracteres estudados. Ao término do presente estudo, foi constatado que a aplicação de doses crescentes de $\mathrm{N}$ e K, e a interação NxP afetam os teores de ambos os nutrientes no solo, o sistema radicular e a parte aérea, influenciando, desse modo, sobre o crescimento inicial das plantas de pitaia. Os rendimentos mais satisfatórios ocorrem quando há $0,7 \mathrm{mmol} \mathrm{dm}^{-3} \mathrm{de} \mathrm{K}$ no solo, e 20 - $25 \mathrm{~g} \mathrm{~kg}^{-1}$ de $\mathrm{N}$ com $30-40 \mathrm{~g} \mathrm{~kg}^{-1}$ de $\mathrm{K}$ na parte aérea. Esses valores foram obtidos para a aplicação de $300-450 \mathrm{mg} \mathrm{dm}^{-3}$ de $\mathrm{N}$ com $150-225 \mathrm{mg} \mathrm{dm}^{-3}$ de K. O somatório do comprimento dos cladódios (SCC) e a massa seca do sistema radicular (MSSR) são as variáveis explicativas que apresentam maiores efeitos diretos sobre a massa seca dos cladódios (MSC).

Termos para indexação: adubação, Cactaceae, frutífera exótica, Hylocereus undatus.

\section{EFFECT OF COMBINATIONS OF NITROGEN AND POTASSIUM ON THE GROWTH OF DRAGON FRUIT (Hylocereus undatus)}

\begin{abstract}
Pitaya is still a culture lacking of research and information that support the definition/ adaptation of production systems adapted to Brazilian soils and climate, although in recent years there has been an increase in its agricultural expansion, as in Brazil as in other countries. Thus, the aim of this study was to evaluate the effect of mineral fertilization on the early growth of pitaya and then to obtain directed information to the definition of adequate fertilization managements of the culture. For this purpose, we tested five doses of nitrogen $(\mathrm{N})\left(0,150,300,450\right.$ and $\left.600 \mathrm{mg} \mathrm{dm}^{-3}\right)$ and potassium $(\mathrm{K})(0,75,150,225$ and $300 \mathrm{mg} \mathrm{dm}^{-3}$ ), and the levels of each nutrient, arranged in a $5 \times 5$ factorial design with four blocks. For a significant interaction $(p<0.05)$ it was proceeded an adjustment in response surfaces of type $Y=\beta_{0}+\beta_{1}(A)$ $+\beta_{2}(\mathrm{~A})^{2}+\beta_{3}(\mathrm{~B})+\beta_{4}(\mathrm{~B})^{2}+\beta_{5}(\mathrm{~A})$. (B) + e. Estimates of phenotypic correlations were obtained considering only the range of $\mathrm{N}$ and $\mathrm{K}$ that promoted the best answers to the access pitaya traits. At the end of this study it was found that the application of increasing doses of $\mathrm{N}$ and $\mathrm{K}$, and the interaction $\mathrm{NxP}$ affected the levels of both nutrients in the soil, roots and shoots, thus influencing the early growth of the pitaya plants. The most satisfactory yields occur when there is $0.7 \mathrm{mmol} \mathrm{dm}{ }^{-3}$ of $\mathrm{K}$ in the soil, and $20-25 \mathrm{~g} \mathrm{~kg}^{-1}$ of N with $30-40 \mathrm{~g} \mathrm{~kg}^{-1}$ of $\mathrm{K}$ in the shoot. These values were obtained for the application $300-450 \mathrm{mg} \mathrm{dm}^{-3}$ of N with $150-225 \mathrm{mg} \mathrm{dm}^{-3}$ of $\mathrm{K}$. The sum of the length of cladodes (SCC) and the dry mass of the root system (DMRS) are the explanatory variables that have large direct effects on the dry weight of cladodes (DWC). Index terms: manuring, Cactaceae, exotic fruits, Hylocereus undatus.

\footnotetext{
${ }^{1}$ (Trabalho 296-13) Recebido em: 22-08-2013. Aceito para publicação: 23-04-2014.

${ }^{2}$ Doutorando no Programa de Pós-Graduação em Agronomia: Fitotecnia, Campus do Pici, (UFC). E-mail: edmilson_i@hotmail.com ${ }^{3}$ Prof. Adjunto do Dept ${ }^{\circ}$ de Fitotecnia á (UFC), Campus do Pici. E-mail: mcleber@ufc.br

${ }^{4}$ Pesquisador da Embrapa Agroindústria Tropical, Fortaleza-CE. E-mail: lindberg@ embrapa.br

${ }^{5}$ Graduando em Agronomia na Universidade Federal Rural do Semi-Árido (UFERSA). E-mail: neimararcanjo@yahoo.com.br

${ }^{6}$ Prof. Adjunto do Dept ${ }^{\circ}$ de Fitotecnia (UFC), Campus do Pici. E-mail: juliodovale@ufc.br
} 


\section{INTRODUÇ̃̃O}

A pitaia (Hylocereus undatus) é uma cactácea epífita, perene, suculenta, que apresenta caule do tipo cladódio, de onde partem numerosas raízes adventícias que permitem o crescimento da planta em árvores e pedras situadas em ambientes sombreados de florestas tropicais da América. A pitaia está distribuída na Costa Rica, Venezuela, Panamá, Uruguai, Brasil, Colômbia e México, sendo os dois últimos países os principais produtores mundiais (DONADIO, 2009; ORTIZ-HERNÁNDEZ; CARRILLO-SALAZAR, 2012).

A produtividade média da pitaia oscila de 10 a $30 \mathrm{t} \mathrm{ha}^{-1}$, o que ocorre em função das condições edafoclimáticas, técnicas de cultivo e idade do pomar (LE BELLEC; VAILLANT; INBERT, 2006). No Brasil, a região Sudeste é a principal produtora, com destaque à cidade de Catanduva (SP), que é uma das pioneiras no cultivo de pitaia no País e, atualmente, apresenta o maior rendimento nacional. Nessa região, a cultura aclimatou-se muito bem, e a produção abrange os meses de dezembro a maio, com produtividade média anual de 14 toneladas de frutos por hectare (BASTOS et al., 2006).

No Ceará, a produção está concentrada na Chapada do Apodi, mais precisamente nos municípios de Limoeiro do Norte e Quixeré. As áreas de cultivo são recentes e estão em fase de definição quanto ao manejo agronômico e à rentabilidade da exploração comercial. As plantas produzem frutos durante o ano inteiro, com pequeno decréscimo nos meses mais chuvosos (janeiro a abril). A safra é comercializada a preços elevados nas principais redes de supermercados de Fortaleza (CE) e/ou exportada para países europeus (NUNES et al., 2014).

Apesar de a pitaia ser uma planta rústica, que se adapta a várias condições edafoclimáticas, a cultura requer o suprimento equilibrado de nutrientes para crescer e produzir satisfatoriamente (MOREIRA et al., 2011; CORRÊA et al., 2014). As funções desempenhadas individualmente por potássio (MARENCO; LOPES, 2011) e nitrogênio (LUDERS, 2004), em seu ciclo vital, são conhecidas; no entanto, o entendimento dos fenômenos que visam a estudar a interação de ambos os nutrientes sobre os processos de absorção, transporte, redistribuição e metabolismo necessitam ser mais bem analisados cientificamente.

Dada a necessidade de estudos a serem realizados com pitaia no Brasil, principalmente no Estado do Ceará, onde o seu cultivo está se expandindo e apresenta elevado potencial, principalmente em virtude da produção de frutos durante o ano inteiro, realizou-se um experimento com o objetivo de compreender os efeitos de combinações de doses de $\mathrm{N}$ e $\mathrm{K}$ sobre a disponibilidade desses nutrientes no solo, sistema radicular e parte aérea, assim como a influência dessas relações sobre o crescimento de mudas de pitaia.

\section{MATERIAL E MÉTODOS}

O experimento foi conduzido no período de outubro de 2011 a março de 2012, em casa de vegetação, com irradiação de $550 \mu \mathrm{mol}$ fótons $\mathrm{m}^{-2} \mathrm{~s}^{-1}$, localizada no Setor de Horticultura do Departamento de Fitotecnia, Centro de Ciências Agrárias da Universidade Federal do Ceará (UFC), Fortaleza-CE ( $3^{\circ} 43^{\prime} 02^{\prime \prime}$ de latitude S e $38^{\circ} 32^{\prime} 35^{\prime \prime}$ de longitude WGr; altitude de 19,6 m) (INSTITUTO DE PESQUISA E ESTRATÉGIA ECONÔMICA DO CEARÁ, 2011). De acordo com a classificação de Köppen (1918), o clima é do tipo Aw'. Trata-se de uma região pertencente ao grupo de clima tropical chuvoso, com temperatura média anual de $26,5^{\circ} \mathrm{C}$.

O plantio dos cladódios de pitaia (Hylocereus undatus) foi realizado em vasos com capacidade para $12 \mathrm{dm}^{3}$, preenchidos com $10 \mathrm{dm}^{3}$ de solo. $\mathrm{O}$ solo foi amostrado e encaminhado ao laboratório, onde foi analisado quanto às suas características físicas e químicas. Ele apresentou as seguintes características: classificação textural - areia franca; composição química - pH (água): 7,2; Ca, Mg, K, Na, $\mathrm{Al}^{3+}, \mathrm{H}+$ $\mathrm{Al}$, respectivamente, 0,$40 ; 0,30 ; 0,20 ; 0,10 ; 0,0$ e 0,0 $\mathrm{mmol}_{\mathrm{c}} \mathrm{dm}^{-3} ; \mathrm{P}, \mathrm{Cu}, \mathrm{Fe}, \mathrm{Zn}$ e $\mathrm{Mn}$, respectivamente, 18,$0 ; 0,50 ; 30,16 ; 2,48$ e $2,26 \mathrm{mg} \mathrm{dm}^{-3}$; e matéria orgânica de $1,80 \mathrm{~g} \mathrm{~kg}^{-1}$.

Como materiais propagativos, utilizaram-se cladódios com três classes de comprimento: pequeno $(8-20 \mathrm{~cm})$, médio $(21-33 \mathrm{~cm})$ e grande $(34-46 \mathrm{~cm})$, havendo a distribuição de, pelo menos, dois cladódios de tamanho médio dentre as quatro repetições utilizadas para cada tratamento. A irrigação foi realizada manualmente. Para a manutenção do teor de umidade do solo, em torno de $60 \%$, foram utilizados béqueres contendo as quantidades de água calculadas. Por sua vez, o tutoramento dos vasos foi realizado aos 95 dias, tendo em vista o acentuado crescimento das plantas, a partir desse período.

Em virtude da ausência de recomendação de adubação para a cultura da pitaia, as doses foram estimadas com base em recomendação geral para experimento em vasos, boletins técnicos da cultura do abacaxi e experimentos conduzidos com palma forrageira. Foram testadas cinco doses de $\mathrm{N}(0 ; 150$; 300; 450 e $600 \mathrm{mg} \mathrm{dm}^{-3}$ de N) e cinco doses de $\mathrm{K}$ $\left(0 ; 75 ; 150 ; 225\right.$ e $300 \mathrm{mg} \mathrm{dm}^{-3}$ de $\left.\mathrm{K}\right)$, sendo que os níveis de ambos os nutrientes foram arranjados 
em esquema fatorial $5 \times 5$, com quatro repetições. Os adubos utilizados como fonte de $\mathrm{N}$ e $\mathrm{K}$ foram, respectivamente, ureia $(45 \%$ de $\mathrm{N})$ e cloreto de potássio $\left(60 \%\right.$ de $\left.\mathrm{K}_{2} \mathrm{O}\right)$. Para a adubação básica (igual em todos os vasos), foram utilizados $832 \mathrm{mg} \mathrm{dm}^{-3} \mathrm{de}$ superfosfato simples e $27,7 \mathrm{mg} \mathrm{dm}^{-3}$ de FTE BR-12. À exceção do superfosfato simples e do FTE BR-12, que foram aplicados em quantidade total, durante $\mathrm{O}$ preenchimento dos vasos, os demais adubos foram adicionados em cinco vezes iguais, solubilizados em água para favorecer sua incorporação ao solo.

Aos 180 dias do plantio dos cladódios (cerca de 150 dias após a aplicação dos tratamentos), fizeram-se a análise vegetativa das plantas, a coleta dos cladódios laterais e a amostragem do solo utilizado para o preenchimento dos vasos. Os caracteres vegetativos avaliados foram: somatório do comprimento dos cladódios (SCC), número de cladódios (NC), massa seca dos cladódios (MSC), comprimento do sistema radicular (CSR) e massa seca do sistema radicular (MSSR). O material foi moído, para a determinação dos teores de $\mathrm{N}$ e $\mathrm{K}$ na parte aérea. Em cada vaso, o solo foi coletado, embalado em saco plástico, identificado e encaminhado para o laboratório, onde foi submetido à secagem em estufa com circulação forçada de ar, à temperatura de $50^{\circ} \mathrm{C}$, durante 24 horas. Em seguida, o material foi peneirado e analisado quanto ao teor de K.

Os resultados das variáveis químicas e vegetativas foram submetidos à análise de variância (teste $\mathrm{F}$ com nível de significância $\mathrm{p}<0,05$ ), de modo a avaliar os efeitos principais de cada uma das variáveis (A e B) e da interação (A x B). Para a interação significativa $(\mathrm{p}<0,05)$, procedeu-se ao ajuste em superfícies de resposta do tipo $\mathrm{Y}=\beta_{0}+$ $\beta_{1}(\mathrm{~A})+\beta_{2}(\mathrm{~A})^{2}+\beta_{3}(\mathrm{~B})+\beta_{4}(\mathrm{~B})^{2}+\beta_{5}(\mathrm{~A}) .(\mathrm{B})+e, \mathrm{em}$ que $Y$ foi a variável de resposta; $A$, dose de $A$ (mg $\mathrm{dm}^{-3}$ de A); $\mathrm{B}$, dose de B (mg dm $\mathrm{dm}^{-3}$ de B); $\beta_{0}, \beta_{1}$, $\beta_{2}, \beta_{3}, \beta_{4}$ e $\beta_{5}$, os parâmetros do modelo e $e$, o erro aleatório associado a cada observação. Após este ajuste inicial, a significância de cada parâmetro foi testada a $\mathrm{p}<0,05$.

As estimativas das correlações vegetativas foram obtidas considerando apenas o intervalo das doses de $\mathrm{N}$ e $\mathrm{K}$ que promoveram as melhores respostas do acesso de pitaia para os caracteres estudados. Posteriormente, foi realizada a diagnose de multicolinearidade entre os caracteres explicativos, e seu grau na matriz de correlação foi estabelecido com base no número de condição. $\mathrm{O}$ desdobramento das correlações fenotípicas, em efeitos diretos e indiretos dos caracteres explicativos sobre a massa seca de cladódios (caráter dependente), foi feito por meio da análise de trilha (WRIGHT, 1921). As análises estatísticas e de trilhas foram executadas por meio do aplicativo computacional em Genética e Estatística, GENES.

\section{RESULTADOS E DISCUSSÃO}

A interação nitrogênio-potássio foi significativa $(\mathrm{p}<0,01)$ para o teor de $\mathrm{K}$ no solo, teores de $\mathrm{N}$ e $\mathrm{K}$ na parte aérea e para as variáveis vegetativas analisadas. Em razão disso, as médias das respectivas variáveis foram analisadas através de superfícies de resposta com modelo matemático do tipo $\mathrm{Y}=\beta_{0}+\beta_{1}(\mathrm{~N})+\beta_{2}(\mathrm{~N})^{2}+\beta_{3}(\mathrm{~K})+\beta_{4}(\mathrm{~K})^{2}+$ $\beta_{5}(\mathrm{~N})$. ( K $)+e$.

O teor de $\mathrm{K}$ no solo oscilou de 0,13 a 2,89 $\mathrm{mmol}_{\mathrm{c}} \mathrm{dm}^{-3}$. Os maiores valores $\left(>1,40 \mathrm{mmol}_{\mathrm{c}} \mathrm{dm}^{-3}\right)$ foram resultantes da aplicação de $0-150 \mathrm{mg} \mathrm{dm}^{-3}$ de $\mathrm{N}$ com 225 - $300 \mathrm{mg} \mathrm{dm}^{-3}$ de K. Esses resultados demonstraram que a aplicação de doses elevadas de $\mathrm{N}\left(300-600 \mathrm{mg} \mathrm{dm}^{-3} \mathrm{de} \mathrm{N}\right)$ reduziu a disponibilidade de K no solo (Figura 1A). Possivelmente, a utilização dessas doses de $\mathrm{N}$ estimulou o incremento vegetativo da planta (SILVA et al., 2012; BREDEMEIER; MUNDSTOCK, 2000) e, consequentemente, houve aumento na demanda da planta por $\mathrm{K}$, exaurindo a disponibilidade de K na solução do solo.

$\mathrm{O}$ teor de $\mathrm{K}$ na parte aérea variou de 12,87 a $46,00 \mathrm{~g} \mathrm{~kg}^{-1}$. As maiores médias $\left(>30,00 \mathrm{~g} \mathrm{~kg}^{-1}\right)$ foram encontradas com a aplicação de $150-300 \mathrm{mg} \mathrm{dm}^{-3}$ de N com 225 - $300 \mathrm{mg} \mathrm{dm}^{-3}$ de K (Figura 1B). Estas combinações que resultaram nos maiores teores de $\mathrm{K}$ na parte aérea, simultaneamente, possibilitaram maior disponibilidade de $\mathrm{K}$ no solo $(0,70-1,40 \mathrm{~g}$ $\mathrm{kg}^{-1}$ ) (Figura 1A). Esses resultados corroboraram os estudos da interação nitrogênio-potássio realizados por Rodrigues et al. (2010) e Lacoeuilhe e Gicquiaux (1971). De acordo com esses autores, a interação é bastante influenciada pela disponibilidade de $\mathrm{K}$ no solo. Em solos com teores de $\mathrm{K}$ considerados altos, o $\mathrm{N}$ estimula o crescimento vegetativo, aumentando-se o teor de K na parte aérea e a utilização das reservas desse nutriente na solução do solo.

$\mathrm{O}$ teor de $\mathrm{N}$ na parte aérea oscilou de 3,36 a $30,40 \mathrm{~g} \mathrm{~kg}^{-1}$. Os maiores valores $\left(>25,00 \mathrm{~g} \mathrm{~kg}^{-}\right.$ $\left.{ }^{1}\right)$ foram resultantes da adição de $450-600 \mathrm{mg}$ $\mathrm{dm}^{-3}$ de $\mathrm{N}$ com $0-75 \mathrm{mg} \mathrm{dm}{ }^{-3}$ de K (Figura 1D). Observou-se, portanto, que o aumento no acúmulo de $\mathrm{N}$ na parte aérea foi induzido pela aplicação de doses crescentes de N. A combinação entre a maior dose $\mathrm{N}$ e a supressão da aplicação de $\mathrm{K}$ resultou num incremento de $27,04 \mathrm{~g} \mathrm{~kg}^{-1}$ de $\mathrm{N}$ na parte aérea, comparativamente à média da testemunha. Embora tenha ocorrido significativo incremento, 
possivelmente, houve menor eficiência de uso do N, induzida pela deficiência de K. Conforme Sarmento et al. (2008), quando o suprimento de $\mathrm{N}$ é realizado em condições de baixa disponibilidade de $\mathrm{K}$, há um favorecimento para o acúmulo de compostos de $\mathrm{N}$ solúvel e, consequentemente, ocorre baixa eficiência de uso do N pela planta.

O SCC variou de 9,00 a 188,75 cm. As maiores médias $(>150,00 \mathrm{~cm})$ foram oriundas da adição de 300 - $600 \mathrm{mg} \mathrm{dm}^{-3}$ de $\mathrm{N}$ com 75 - $300 \mathrm{mg}$ $\mathrm{dm}^{-3}$ de K (Figura 2A). Esses resultados coincidiram com o aumento da disponibilidade de $\mathrm{N}$ e $\mathrm{K}$ no solo $\left(0,70-1,40 \mathrm{mmol} \mathrm{dm}^{-3}\right)$ (Figura 1A), elevado acúmulo de $\mathrm{N}$ na parte aérea $\left(20,00-25,00 \mathrm{~g} \mathrm{~kg}^{-1} \mathrm{de}\right.$ $\mathrm{N})$ (Figura 1C) e teor intermediário a elevado de $\mathrm{K}$ na parte aérea $\left(30,00-40,00 \mathrm{~g} \mathrm{~kg}^{-1}\right.$ de K) (Figura 1B), corroborando a hipótese de que a aplicação de doses satisfatórias de K aumenta a eficiência de uso do N pela planta (LACOEUILHE; GICQUIAUX, 1971).

$\mathrm{O}$ NC variou de 2,00 a 11,00 unidades. Os maiores valores ( $>8,00$ unidades) foram encontrados com a adição de $300-450 \mathrm{mg} \mathrm{dm}^{-3}$ de $\mathrm{N}$ com 75 - $225 \mathrm{mg} \mathrm{dm}^{-3}$ de K (Figura 2B). Constatouse, portanto, que as maiores médias de $\mathrm{NC}$ foram verificadas quando as doses de $\mathrm{N}$ e $\mathrm{K}$ aplicadas ao solo permitiram o aumento da disponibilidade de ambos os nutrientes na solução do solo $(0,70-1,40$ mmol dm ${ }^{-3}$ de K) (Figura $\left.1 \mathrm{~A}\right)$, bem como médio a elevado acúmulo de $\mathrm{N}\left(20-25 \mathrm{~g} \mathrm{~kg}^{-1}\right.$ de $\left.\mathrm{N}\right)$ e $\mathrm{K}(30$ $-40 \mathrm{~g} \mathrm{~kg}^{-1}$ de K) na parte aérea (Figuras $1 \mathrm{~A} \mathrm{e} 1 \mathrm{~B}$ ).

A massa seca dos cladódios variou de 5,00 $-43,75 \mathrm{~g}$. Os menores valores $(<20,00 \mathrm{~g})$ foram evidenciados para a aplicação de $0-150 \mathrm{mg} \mathrm{dm}^{-3} \mathrm{de}$ $\mathrm{N}$ com $0-300 \mathrm{mg} \mathrm{dm}^{-3}$ de $\mathrm{K}$, enquanto os maiores resultados $(>30,00 \mathrm{~g})$ foram obtidos com a utilização de $300-600 \mathrm{mg} \mathrm{dm}^{-3}$ de $\mathrm{N}$ e $75-300 \mathrm{mg} \mathrm{dm}^{-3}$ de $\mathrm{K}$ (Figura 2D). Esses resultados deixaram claro que o $\mathrm{N}$ exerce efeito limitante sobre o aumento de MSC e das demais variáveis vegetativas estudadas, enquanto o $\mathrm{K}$ teve efeito mais evidenciado para o aumento da eficiência de uso de $\mathrm{N}$ pela planta. Houve incremento de até oito vezes na MSC, exprimindo a importância da aplicação racional de $\mathrm{N}$ e K sobre o crescimento inicial de pitaia. Este aumento do NC foi quantitativamente semelhante àqueles obtidos por Dubeux Júnior et al. (2010) para os artículos de mudas de palma forrageira.

Observou-se que a MSC variou de 5,00 a 43,75 g, enquanto a MFC oscilou de 23,75 a 356,25 g (Figuras 2C e 2D). Relacionando-se ambos os resultados, estimou-se que torno de 79,00$88,00 \%$ do tecido vegetal estava constituído por água, corroborando o teor relativo de umidade recomendado por Goldstein et al. (1991) para cactos suculentos.

O comprimento do sistema radicular variou de 35,00 a $82,50 \mathrm{~cm}$. Os menores valores $(<50,00$ $\mathrm{cm})$ foram constatados com a utilização de 450 - 600 $\mathrm{mg} \mathrm{dm} \mathrm{m}^{-3}$ de $\mathrm{N}$ e $150-300 \mathrm{mg} \mathrm{dm}^{-3}$ de K (Figura 2E). Logo, observou-se que a utilização de elevadas doses de $\mathrm{N}$ e K ocasionaram redução do crescimento vertical do sistema radicular, concomitantemente ao estímulo do incremento de parte aérea, o que se enquadra com os estudos de Silva (2012). Conforme esse autor, o excesso de $\mathrm{N}$ no solo pode proporcionar o aumento da relação parte aérea/raiz, reduzindo o crescimento longitudinal do sistema radicular e, consequentemente, diminuindo a capacidade de resistência das plantas a períodos secos.

A massa seca do sistema radicular oscilou de 9,50 a $17,50 \mathrm{~g}$. Os menores resultados $(<12,00$ g) foram alcançados para a aplicação de 450 - 600 mg dm ${ }^{-3}$ de $\mathrm{N}$ com 0 - $75 \mathrm{mg} \mathrm{dm}^{-3}$ de $\mathrm{K}$; ou $0-600$ $\mathrm{mg} \mathrm{dm}^{-3}$ de $\mathrm{N}$ com $300 \mathrm{mg} \mathrm{dm}^{-3}$ de K (Figura 2F). Por sua vez, os maiores valores $(>13,00 \mathrm{~g})$ foram obtidos para a adição de $0-450 \mathrm{mg} \mathrm{dm}^{-3}$ de $\mathrm{N}$ com $75-225 \mathrm{mg} \mathrm{dm}^{-3}$ de K (Figura 2F). Os resultados apresentaram que a deficiência de $\mathrm{N}$ e $\mathrm{K}$ no solo culminou em aumento da massa seca do sistema radicular. Certamente, o uso de elevadas doses de $\mathrm{N}$ e K estimulou o crescimento vegetativo, interferindo negativamente sobre o incremento do sistema radicular.

Para Cabral et al. (2011), o estudo de correlações é uma medida de associação e não permite concluir sobre o estudo da relação de causa e efeito. Silva et al. (2005) acrescentam que a análise de trilha proporciona o detalhamento dos efeitos diretos e indiretos das influências dos caracteres envolvidos, complementando, assim, as informações dadas pela correlação. Com o intuito de se conhecer os efeitos diretos e indiretos dos caracteres sobre a variável básica, massa seca dos cladódios, procedeu-se à análise de trilha.

Para reduzir os efeitos de multicolinearidade e, desse modo, a atenuação de conclusões imprecisas, a análise de trilha foi construída, apenas com as combinações de doses de $\mathrm{N}$ e K, que apresentaram os resultados mais satisfatórios, em termos de disponibilidade dos elementos no solo e no tecido vegetal, e de incremento das variáveis vegetativas avaliadas. De acordo com Coimbra et al. (2005), entre os efeitos peculiares de uma elevada multicolinearidade, podem ser citados: estimativa inconsistente do coeficiente de regressão e também uma superestimativa dos efeitos diretos das variáveis explicativas sobre a variável resposta, o que pode levar à interpretação equivocada. 
Em resumo, as doses de $\mathrm{N}$ e $\mathrm{K}$ utilizadas para a execução da análise de trilha foram as seguintes: 300 - $450 \mathrm{mg} \mathrm{dm}^{-3}$ de $\mathrm{N}$ com 150 - $225 \mathrm{mg} \mathrm{dm}^{-3}$ de $\mathrm{K}$, as quais resultaram em quatro combinações nitrogênio-potássio (Figuras 1 e 2). As variáveis, teor de $\mathrm{K}$ no solo e MFC, também foram excluídas da análise de trilha devido à mesma justificativa estatística (reduzir os efeitos de multicolinearidade). A interação nitrogênio-potássio foi significativa a $(p<0,05)$, pelo teste $\mathrm{F}$, para os teores de $\mathrm{N}$ e $\mathrm{K}$ na parte aérea, NC, MSC e CSSR. O SCC foi influenciado pela aplicação de doses de $\mathrm{N}$ e K, individualmente, enquanto a MSSR não sofreu efeito da adição de ambos os nutrientes (Tabela 1).

O coeficiente de determinação obtido no presente estudo foi de $98,50 \%$, enquanto o efeito residual foi de $12,10 \%$. Os valores dos coeficientes de variação oscilaram de 6,30 - 25,20\%, o que indica boa precisão experimental. A variável básica, MSC, foi correlacionada positivamente aos teores de $\mathrm{Ne}$ K na parte aérea, SCC, NC e MSSR. Não obstante, o CSSR correlacionou-se negativamente à MSC, indicando que o aumento vertical do sistema radicular ocasionou redução na relação parte aérea-raiz. Esses resultados corroboraram aqueles apresentados nas superfícies de resposta expostas nas Figuras 1 e 2 . O SCC e a MSSR foram as variáveis explicativas que apresentaram maiores efeitos positivos e diretos sobre a MSC.

Observou-se que o teor de $\mathrm{K}$ na parte aérea foi correlacionado em 58,20\% à variável básica, dos quais, $21,90 \%$ foram efeitos indiretos via SCC. O teor de $\mathrm{N}$ na parte aérea foi correlacionado em $18,40 \%$ à MSC, dos quais $17,40 \%$ foram resultantes de efeito direto dessa variável explicativa sobre a variável básica MSC. O SCC correlacionou-se em 97,10\% à MSC, onde apenas 28,70\% da correlação ocorreram devido a efeitos diretos. O NC correlacionou-se em $55,80 \%$ à MSC, sendo que a maior parte dos efeitos de associação $(21,30 \%)$ foram efeitos indiretos via SCC.

O CSSR correlacionou-se negativamente, em 97,20\%, à variável básica, e os efeitos indiretos e negativos via SCC foram os mais expressivos $(28,70 \%)$ (Tabela1). Segundo Kurek et al. (2001), uma correlação negativa indica que o aumento de uma variável corresponde à diminuição da outra. Esses autores acrescentam, ainda, que este fato pode ser atribuído à competição intrínseca da planta por fotoassimilados e aos fatores ambientais.

Embora o CSSR tenha exprimido efeito negativo sobre a MSC, a MSSR apresentou correlação positiva de $95,20 \%$ à variável básica. A maior parte destes efeitos foram diretos e estimados em 32,30\% (Tabela 1). Possivelmente, isso ocorreu devido ao maior crescimento lateral das raízes, estimulado pela boa disponibilidade de nutrientes na camada menos profunda do solo. Por sua vez, houve redução do comprimento vertical do sistema radicular e aumento da relação parte aérea-raiz. Portanto, a aplicação de doses crescentes de N e K resultou em efeitos contrários na relação parte aérearaiz, culminando em efeito negativo do CSSR sobre a variável básica. 

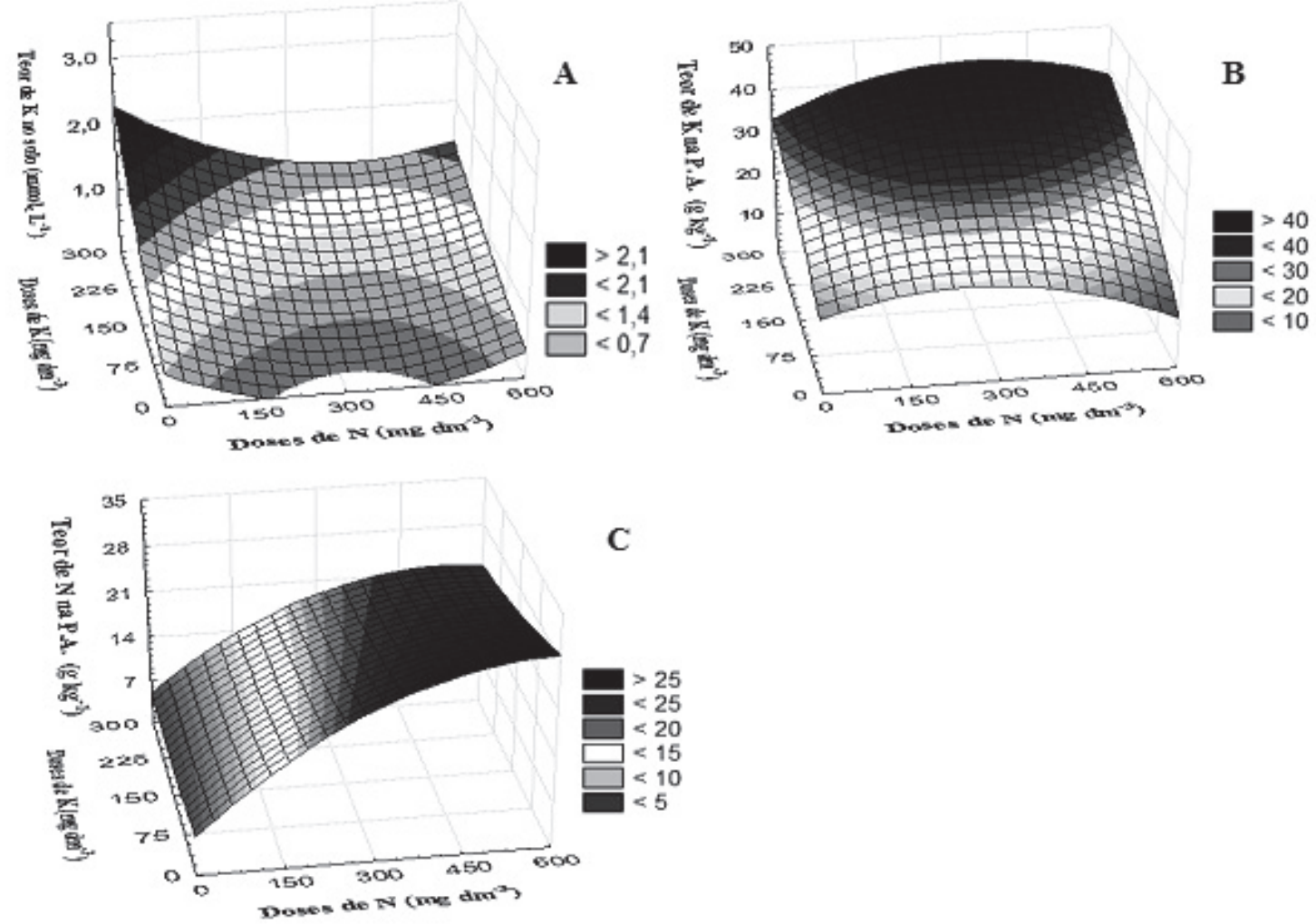

FIGURA 1 - Influência de doses de $\mathrm{N}$ e K sobre o teor de ambos os nutrientes em plantas de pitaia com 180 dias de idade. 1A: teor de $\mathrm{K}$ no solo $\left[\hat{\mathrm{Y}}=0,4939-0,0040(\mathrm{~N})+0,000006375(\mathrm{~N})^{2}+0,0046(\mathrm{~K})\right.$ $\left.+0,0000042159(\mathrm{~K})^{2}-0,000004471(\mathrm{~N})(\mathrm{K}) ; \mathrm{R}^{2}=0,6270^{*}\right]$; 1B: teor de $\mathrm{K}$ na parte aérea $[\hat{\mathrm{Y}}$ $=17,6935+0,0422(\mathrm{~N})-0,000086406(\mathrm{~N})^{2}+0,0865(\mathrm{~K})-0,0001(\mathrm{~K})^{2}+0,000064676(\mathrm{~N})(\mathrm{K})$; $\left.\mathrm{R}^{2}=0,6899^{*}\right]$; 1C: teor de $\mathrm{N}$ na parte aérea $\left[\hat{\mathrm{Y}}=6,0133+0,0750(\mathrm{~N})-0,000060965(\mathrm{~N})^{2}-\right.$ $\left.0,0140(\mathrm{~K})-0,000035225(\mathrm{~K})^{2}-0,000035413(\mathrm{~N})(\mathrm{K}) ; \mathrm{R}^{2}=0,8500^{*}\right]$. Universidade Federal do Ceará. Fortaleza-CE, 2013. 

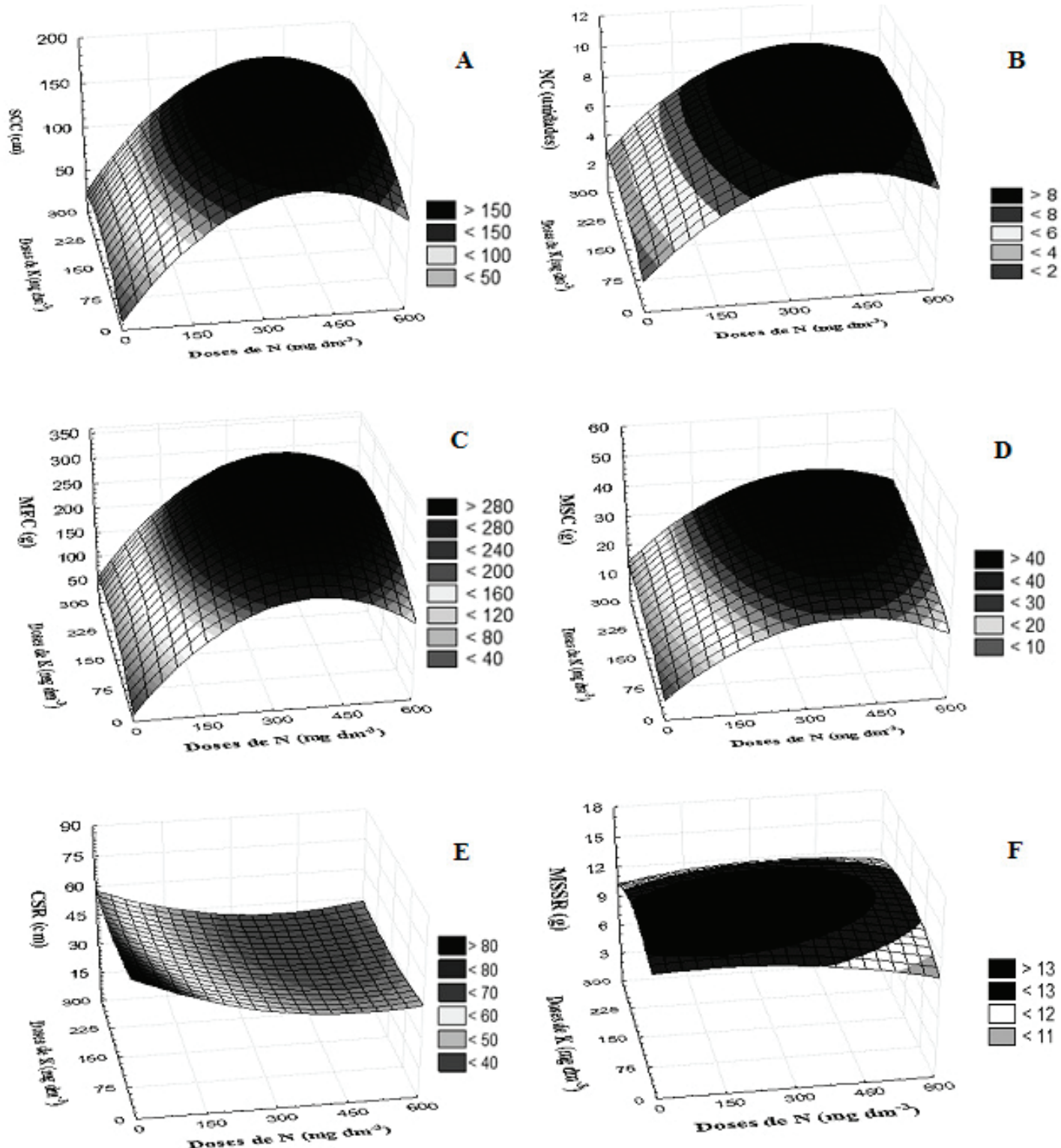

FIGURA 2 - Influência de doses de N e K sobre as características vegetativas de plantas de pitaia com 180 dias de idade. 2A: somatório do comprimento dos cladódios $\left[9,9086+0,6696(\mathrm{~N})-0,0009(\mathrm{~N})^{2}\right.$ $\left.+0,3082(\mathrm{~K})-0,0009(\mathrm{~K})^{2}+0,0001(\mathrm{~N})(\mathrm{K}) ; \mathrm{R}^{2}=0,4612 *\right] ; \mathbf{2 B}$ : número de cladódios $[\hat{\mathrm{Y}}=$ $2,0914+0,0307(\mathrm{~N})-0,000038254(\mathrm{~N})^{2}+0,0119(\mathrm{~K})-0,000031746(\mathrm{~K})^{2}+0,0000037778(\mathrm{~N})$ $\left.(\mathrm{K}) ; \mathrm{R}^{2}=0,5150^{*}\right] ; \mathbf{2 C}$ : massa fresca dos cladódios $\left[\hat{\mathrm{Y}}=15,4071+1,0659(\mathrm{~N})-0,0014(\mathrm{~N})^{2}\right.$ $\left.+0,66(\mathrm{~K})-0,0018(\mathrm{~K})^{2}+0,0002(\mathrm{~N})(\mathrm{K}) ; \mathrm{R}^{2}=0,4661 *\right] ; 2 \mathrm{D}$ : massa seca dos cladódios $[\hat{\mathrm{Y}}=$ $\left.6,6929+0,1204(\mathrm{~N})-0,0002(\mathrm{~N})^{2}+0,058(\mathrm{~K})-0,0001(\mathrm{~K})^{2}+0,00003889(\mathrm{~N})(\mathrm{K}) ; \mathrm{R}^{2}=0,4296^{*}\right]$; 2E: comprimento do sistema radicular $\left[\hat{\mathrm{Y}}=69,7543+0,1019(\mathrm{~N})+0,0001(\mathrm{~N})^{2}-0,0775(\mathrm{~K})\right.$ $\left.+0,0001(\mathrm{~K})^{2}+0,000048444(\mathrm{~N})(\mathrm{K}) ; \mathrm{R}^{2}=0,5546^{* *}\right] ; \mathbf{2 F}$ : massa seca do sistema radicular $[\hat{\mathrm{Y}}$ $=12,4629+0,0029(\mathrm{~N})-0,000011429(\mathrm{~N})^{2}+0,0178(\mathrm{~K})-0,00008381(\mathrm{~K})^{2}+0,000017333(\mathrm{~N})$ $(\mathrm{K}) ; \mathrm{R}^{2}=0,0421^{*}$. Universidade Federal do Ceará. Fortaleza-CE, 2013. 
TABELA 1 - Estimativas dos efeitos diretos e indiretos, que envolvem a variável principal massa seca de cladódios (MSC) e as variáveis independentes explicativas, teor de potássio (KPA) e nitrogênio na parte aérea (NPA), somatório do comprimento (SCC) e número de cladódios (NC), comprimento (CSSR) e massa seca de raiz (MSSR) mensurada num acesso de pitaia (Hylocereus undatus) avaliado quanto a doses crescentes de nitrogênio e potássio FortalezaCE, Brasil, 2013.

\begin{tabular}{|c|c|c|}
\hline Caractere & Efeitos de Associação & Estimativa \\
\hline \multirow{7}{*}{ Potássio na parte aérea (KPA) } & Direto sobre MSC & 0,095 \\
\hline & Indireto via NPA & $-0,121$ \\
\hline & Indireto via SCC & 0,219 \\
\hline & Indireto via $\mathrm{NC}$ & 0,078 \\
\hline & Indireto via CSSR & 0,208 \\
\hline & Indireto via MSSR & 0,099 \\
\hline & Total & 0,582 \\
\hline \multirow{7}{*}{ Nitrogênio na parte aérea (NPA) } & Direto sobre MSC & 0,175 \\
\hline & Indireto via KPA & $-0,066$ \\
\hline & Indireto via SCC & $-0,017$ \\
\hline & Indireto via $\mathrm{NC}$ & $-0,056$ \\
\hline & Indireto via CSSR & $-0,014$ \\
\hline & Indireto via MSSR & 0,153 \\
\hline & Total & 0,184 \\
\hline \multirow{7}{*}{ Somatório do comprimento de cladódios (SCC) } & Direto sobre MSC & 0,287 \\
\hline & Indireto via KPA & 0,072 \\
\hline & Indireto via NPA & $-0,010$ \\
\hline & Indireto via $\mathrm{NC}$ & 0,058 \\
\hline & Indireto via CSSR & 0,275 \\
\hline & Indireto via MSSR & 0,275 \\
\hline & Total & 0,971 \\
\hline \multirow{7}{*}{ Número de cladódios (NC) } & Direto sobre MSC & 0,078 \\
\hline & Indireto via KPA & 0,095 \\
\hline & Indireto via NPA & $-0,125$ \\
\hline & Indireto via $\mathrm{SCC}$ & 0,213 \\
\hline & Indireto via CSSR & 0,203 \\
\hline & Indireto via MSSR & 0,090 \\
\hline & Total & 0,558 \\
\hline \multirow{7}{*}{ Comprimento do sistema radicular (CSSR) } & Direto sobre MSC & $-0,275$ \\
\hline & Indireto via KPA & $-0,072$ \\
\hline & Indireto via NPA & 0,009 \\
\hline & Indireto via SCC & $-0,287$ \\
\hline & Indireto via $\mathrm{NC}$ & $-0,058$ \\
\hline & Indireto via MSSR & $-0,276$ \\
\hline & Total & $-0,972$ \\
\hline \multirow{3}{*}{ Massa seca de raiz (MSSR) } & Direto sobre MSC & 0,323 \\
\hline & Indireto via KPA & 0,029 \\
\hline & Indireto via NPA & 0,083 \\
\hline \multirow{3}{*}{ Massa seca de raiz (MSSR) } & $\begin{array}{l}\text { Indireto via SCC } \\
\text { Indireto via NC }\end{array}$ & $\begin{array}{l}0,245 \\
0,022\end{array}$ \\
\hline & Indireto via CSSR & 0,235 \\
\hline & Total & 0,952 \\
\hline Coeficiente de determinação & & 0,985 \\
\hline Valor de $k$ usado na análise & & 0,045 \\
\hline Efeito da variável residual & & 0,121 \\
\hline
\end{tabular}




\section{CONCLUSÕES}

A aplicação de N e K, e a interação nitrogêniopotássio afetam a disponibilidade de ambos os nutrientes no solo e na parte aérea, influenciando, assim, o crescimento de mudas de pitaia.

Os rendimentos mais satisfatórios ocorrem quando há $0,7 \mathrm{mmol} \mathrm{dm}^{-3}$ de $\mathrm{K}$ no solo, e $20-25 \mathrm{~g}$ $\mathrm{kg}^{-1}$ de $\mathrm{N}$ com 30 - $40 \mathrm{~g} \mathrm{~kg}^{-1}$ de $\mathrm{K}$ na parte aérea, resultados esses que foram obtidos com a aplicação de $300-450 \mathrm{mg} \mathrm{dm}^{-3}$ de $\mathrm{N}$ com 150 - $225 \mathrm{mg} \mathrm{dm}^{-3}$ de $\mathrm{K}$.

O somatório do comprimento dos cladódios (SCC) e a massa seca do sistema radicular (MSSR) são as variáveis explicativas (independentes) que apresentam maiores efeitos positivos e diretos sobre a massa seca dos cladódios (MSC).

\section{AGRADECIMENTOS}

Agradecemos à CAPES, pelo auxílio financeiro (Projeto 23038.006862/2010-02 - Edital 029/2010).

\section{REFERÊNCIAS}

BASTOS, D. C.; PIO, R.; SCARPARE FILHO, J. A.; LIBARDI, M. N.; ALMEIDA, L. F. P. de; GALUCHI, T. P. D.; BAKKER, S. T. Propagação de pitaiavermelha por estaquia. Ciência e Agrotecnologia, Lavras, v. 30, n.6, p.1106-1109, 2006.

BREDEMEIER, C.; MUNDSTOCK, C. M. Regulação da absorção e assimilação do nitrogênio nas plantas. Ciência Rural, Santa Maria, v. 30, n.2, p. $365-372.2000$.

CABRAL, P. D. S.; SOARES, T. C. B.; LIMA, A. B. P.; SOARES, Y. J. B.; SILVA, J. A. da. Análise de trilha do rendimento de grãos de feijoeiro (Phaseolus vulgaris L.) e seus componentes. Revista Ciência Agronômica, Fortaleza, v. 42, n.1, p.132-138, 2011.

COIMBRA, J. L. M.; BENIN, G.; VIEIRA, E. A.; OLIVEIRA, A. C.; CARVAlHO, F. I. F.; GUIDOLIN, A. F.; SOARES, A. P. Consequências da multicolinearidade sobre a análise de trilha em canola. Ciência Rural, Santa Maria, v. 35, n.2, p.347-352, 2005.
CORRÊA, M. C. M.; ALMEIDA, E. I. B.; MARQUES, V. B.; VALE, J. C.; AQUINO, B. F. Crescimento inicial de pitaia em função de combinações de doses de fósforo-zinco. Revista Brasileira de Fruticultura, Jaboticabal, v. 36, n.1, p. 23-38, 2014.

DONADIO, L.C. Pitaya. Revista Brasileira de Fruticultura, Jaboticabal, v.31, n.3, p.637-929, 2009.

DUBEUX JÚNIOR, J. C. B.; ARAÚJO FILHO, J. T.; SANTOS, M. V. F. dos; LIRA, M. A.; SANTOS, D. C. dos; PESSOA, R. A. S. Adubação mineral no crescimento e composição mineral da palma forrageira-Clone IPA-20. Revista Brasileira de Ciências Agrárias, Recife, v. 5, n.1, p. 129-135. 2010 .

INSTITUTO DE PESQUISA E ESTRATÉGIA ECONÔMICADO CEARÁ. Estatística e Geografia. Lista mantida pelo IPECE. 2011. Online. Disponível

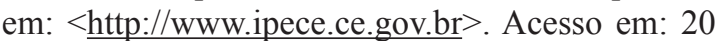
nov. 2012.

KÖPPEN, W. Climatologia: con un estudio de los climas de la tierra. Mexico: Fondo de Cultura Economica, 1918. 478 p.

KUREK, A. J.; CARVALHO, F. I. F. de; ASSMANN, I. C.; MARCHIORO, V. S.; CRUZ, P. J. Análise de trilha como critério de seleção indireta para rendimento de grãos em feijão. Revista Brasileira de Agrociência, Pelotas, v.7, n.1, p. 29-32, 2001.

LACOEUILHE, J. J.; GICQUIAUX, Y. La nutrition en cations de l'ananas à la Martinique. Fruits, Paris, n. 26, p. 519-531. 1971 .

LE BELLEC, F.; VAILLANT, F.; IMBERT, E. Pitahaya (Hylocereus ssp.): a new fruit crop, a market with future. Fruits, Paris, v. 61, p. 237-250. 2006.

LUDERS, L. The pitaya or dragon fruit (Hylocereus undatus). Darwin: University of Darwin, 2004. 5p. (Agnote, 778).

MARENCO, R. A.; LOPES, N. F. Fisiologia vegetal. 3. ed. Viçosa: UFV, 2011. 486p. 
MOREIRA, R. A.; RAMOS, J. D.; MARQUES, V. B.; ARAÚJO, N. A.; MELO, P. C. Crescimento de pitaia-vermelha com adubação orgânica e granulado bioclástico. Ciência Rural, Santa Maria, v. 41, n. 5, p. 785-788, 2011.

NUNES, E. N.; SOUSA, A. S. B. de; LUCENA, C. M. de; SILVA, S. de M.; LUCENA, R. F. P. de; ALVES, C. A. B.; ALVES, R. E. Pitaia (Hylocereus sp.): uma revisão para o Brasil. Gaia Scientia, Areia, v. 8, n.1, p. 90-98. 2014.

ORTIZ-HERNÁNDEZ, Y. D.; CARRILLOSALAZAR, J. A. Pitahaya (Hylocereus spp.): a short review. Comunicata Scientiae, Bom Jesus, v.3, n.4, p.220-237, 2012.

RODRIGUES, A. A.; MENDONÇA, R. M. N.; SILVA, A. P.; SILVA, S. M.; PEREIRA, W. E. Desenvolvimento vegetativo de abacaxizeiros 'Pérola' e 'Smooth Cayenne' no Estado da Paraíba. Revista Brasileira de Fruticultura, Jaboticabal, v. 32, n.1, p.126-134. 2010.
SARMENTO, P.; RODRIGUES, L. R. de A.; CRUZ, M. C. P. da; LUGÃO, S. M. B.; CAMPOS, F. P. de; CENTURION, J. F.; FERREIRA, M. E. Atributos químicos e físicos de um Argissolo cultivado com Panicum maximum Jacq. cv. IPR-86 Milênio, sob lotação rotacionada e adubado com nitrogênio. Revista Brasileira de Ciência do Solo, Viçosa, MG, v.32, n.1, p.183-193. 2008.

SILVA, A. L. P. da; SILVA, A. P. da; SOUZA, A. P. de; SANTOS, D.; SILVA, S. de M.; SILVA, V. B. da. Resposta do abacaxizeiro 'Vitória' a doses de nitrogênio em solos de tabuleiros costeiros da Paraíba. Revista Brasileira de Ciência do Solo, Viçosa, MG, v.36, n.2, p. 447-456, 2012.

SILVA, S. A.; CARVALHO, F. I. F. de; NEDEL, J. L.; CRUZ, P. J.; SILVA, J. A. G. da; CAETANO, V. da R.; HARTWIG, I.; SOUSA, C. da S. Análise de trilha para os componentes de rendimento de grãos em trigo. Bragantia, Campinas, v.64, n.2, p.191196, 2005.

WRIGHT, S. Correlation and causation. Journal of Agricultural Research, Lahore, v. 20, p. 557-585, 1921. 